\title{
La ampliación del tiempo escolar: ¿Se modifican los componentes duros del formato escolar? Revisión bibliográfica sobre estas temáticas
}

\section{School-time Extension: Are the School Structure's Hard Components Modified? Literature Review on the Subject}

\author{
Soledad Vercellino ${ }^{1}$ \\ Sede Atlántica \\ Universidad Nacional de Río Negro \\ Argentina \\ svercellino@unrn.edu.ar
}

Recibido 10 de agosto de 2012 - Corregido 05 de noviembre de 2012 • Aceptado 07 de noviembre de 2012

\begin{abstract}
Resumen. Hace más de dos décadas que asistimos en América Latina y en Argentina, en particular, al desarrollo de diferentes políticas orientadas a ampliar las jornadas escolares. Entendemos que la implementación de estas políticas es una oportunidad para analizar el comportamiento de la organización escolar ante los intentos de modificar uno de sus componentes duros, como lo es el tiempo; y que se constituye en laboratorios naturales que permiten analizar cuánto resiste la organización clásica del tiempo escolar, en qué cambia y cómo esos cambios (de ocurrir) afectan los restantes componentes de lo escolar (espacios, agrupamientos, etc.). Este artículo presenta el estado de la cuestión de los estudios más relevantes en dos campos de indagación en el ámbito de la educación primaria o elemental que intervienen en esta problemática: por un lado, los que refieren al tiempo escolar, su organización y ampliación y; por otro lado, las investigaciones que abordan los componentes estructurales y estructurantes de lo escolar. La revisión bibliográfica realizada nos indica que las investigaciones que han abordado el tiempo escolar y las distintas políticas y programas de extensión de la jornada escolar como objeto de indagación no dan cuenta de las vicisitudes que estas encuentran a la hora de intentar modificar los componentes duros del dispositivo escolar y, por otro lado, que las investigaciones que toman como objeto de estudio el 'dispositivo escolar' no han atendido las distintas experiencias de ampliación del tiempo escolar, aun cuando el tiempo es uno de los elementos constitutivos de dicho dispositivo.
\end{abstract}

Palabras claves. Ampliación de la jornada escolar, tiempo escolar, dispositivo escolar.

\footnotetext{
Licenciada en Psicopedagogía (Universidad Nacional del Comahue). Se desempeña como profesora adjunta regular con dedicación exclusiva (investigadora) de la Sede Atlántica de la Universidad Nacional de Río Negro y asistente de docencia regular de la Universidad Nacional del Comahue, ambas actividades en el área académica de la Metodología de la Investigación. Desarrolla investigaciones en el área de educación. En estos momentos se encuentra ejecutando el proyecto de investigación: "La (re)organización del dispositivo escolar en escuelas primarias de la provincia de Río Negro que implementan un programa de ampliación de la jornada escolar (el Programa Escuelas de Jornada Extendida) y la performatividad del mismo en la relación del alumno con el saber". Dirigido por la Dra. Marta Anadón, Universidad Nacional de Río Negro, Sede Atlántica, aprobado por Res. UNRN № 168/10. Participa como investigadora responsable de Fondos. Asimismo ha depositado su tesis, vinculada a la temática del artículo que se presenta, en la maestría en "Sociedad e Instituciones. Mención en Análisis Institucional", de la Universidad Nacional de San Luis.
} 
URL: http://www.una.ac.cr/educare

\begin{abstract}
For more than two decades we have witnessed in Latin America -in Argentina particularlythe development of policies to expand the school day. We understand that the implementation of such policies is an opportunity to observe the behavior of the school's behavior faced with the attempt to modify one of its hardest components -school-time-; it becomes also a natural laboratory to analyze how much does the traditional organization of school-time can resist, how does it change and how do these changes (if implemented) impact the rest of the school components (spaces, groups, etc.). This paper shows the state of the art of the most significant studies in two research fields, in the context of primary education, on this matter: on the one hand, the studies related to organization and extension of school time and, on the other hand, research on the structural and structuring components of school-related aspects. The literature review indicates that studies on school-time and on the corresponding extension policies and programs do not report the difficulties found when trying to modify the hard components of the school system. Studies with the 'school system' as object of study have not approached the numerous school-time extension experiences, although time is one of the structural elements of the system.
\end{abstract}

Keywords. School-day extension, school time, school system.

Este artículo presenta una revisión bibliográfica sobre los estudios más relevantes en dos campos de indagación en el ámbito de la educación primaria o elemental: por un lado, los que refieren al análisis del tiempo escolar, su organización y las experiencias de ampliación y, por otro, las investigaciones que abordan los componentes estructurales y estructurantes de lo escolar.

La preocupación por la duración del tiempo escolar no es un tema nuevo en el debate sobre la educación, más bien es una preocupación que reconoce argumentaciones sobre la necesidad de incrementar el mismo que datan de fines del siglo XIX (Universidad Nacional de Río negro, 2012). Y desde hace más de dos décadas la ampliación de la jornada escolar se ha erigido como una política educativa privilegiada para la atención, fundamentalmente, de las poblaciones con mayores dificultades de escolarización y de sectores socioeconómicos más desfavorecidos. En esa línea, ampliar el tiempo que los alumnos pasan en la escuela es una de las recomendaciones de política educativa de inicios del siglo XXI de la UNESCO, la que establece como meta alcanzar una jornada de al menos 200 días y al menos 1.000 horas anuales².

En Latinoamérica existen varias experiencias de extensión de la jornada escolar, entre las que cabe citar los centros de experiencia de educación pública (CIEP), creados en los 80 en Río de Janeiro; las escuelas de tiempo completo, implementadas en el Uruguay en la década del 90 y promovidas por el Banco Mundial; las escuelas de tiempo completo implementadas a partir de 1997 en Chile, país que luego decreta el tiempo completo como modalidad para todas las escuelas. Las escuelas bolivarianas, en Venezuela; el Programa Nacional de Escuelas de Tiempo Completo (PNETC), implementado en México desde el 2006. De todas estas experiencias, la de Chile y Uruguay son las más estudiadas (Zorrilla, Langford, Ramírez y García, 2008).

2 La recomendación 7 de la Declaración de Cochabamba de marzo del 2001 también señaló que tal ampliación del horario ha de acompañarse de medidas que faciliten su aprovechamiento efectivo, por lo que es necesario utilizar métodos de enseñanza flexibles y diversificados. 
En el caso de Argentina, la extensión de la jornada escolar ha sido una de las metas educativas impuestas por la Ley N²6.075 de Financiamiento Educativo, la que preveía alcanzar en el año 2010 una oferta de escuelas de jornada extendida o completa para el 30\% de los alumnos de educación básica, partiendo de una base de apenas un 5,5\% de cobertura en el conjunto del país (línea de base 2005). La Ley Nacional de Educación N 26.206 estableció, en su artículo 28, que "las escuelas primarias serán de jornada extendida o completa con la finalidad de asegurar el logro de los objetivos fijados para este nivel".

Un reciente informe (Rivas, Vera y Bezem, 2011) indica que al año 2009 el porcentaje de alumnos en oferta de jornada completa (educación común) ascendía al 5,9\% en todo el país. En este mismo se señala que "el éxito en alcanzar las metas educativas no ha tenido la contundencia de la dimensión financiera, aunque se han logrado avances importantes en algunos aspectos del sistema educativo, [queda] pendiente la meta de extensión de la jornada escolar para los sectores más pobres, una política de esencia redistributiva que todavía no se ha consolidado" (Rivas, Vera y Bezem, 2011, p. 41).

Cabe aclarar que en el país se registran, desde hace más de diez años, programas de ampliación del tiempo escolar, tal es el caso de la Provincia de Buenos Aires, la de Córdoba (con su "Microexperiencia de Extensión de Jornada", luego reformulada como Programa Escuelas de Jornada Ampliada, que abarca el $20 \%$ de las escuelas de la provincia y consiste en una extensión de noventa minutos), Mendoza (Experiencia Piloto de Doble Escolaridad, 2000; Programas de Doble Escolaridad, 2005); Santa Cruz (Programa de Jornada Extendida, 2004); Catamarca y La Pampa con el $16,2 \%$ y el $13,8 \%$, respectivamente de la matrícula escolar estatal en escuelas de jornada completa (Universidad Nacional de Río negro, 2012).

Los distintos programas difieren en lo referido a la cantidad de tiempo que se agrega (un turno más, unas horas más) a la organización curricular: unos priorizan los contenidos "básicos"o "prioritarios" (Lengua y Matemática en primer lugar, y Ciencias Naturales y Sociales, en segundo); otros ponen el acento en áreas "curriculares" y disciplinas ligadas con otras necesidades educativas actuales (Educación Física, Música, Artística, así como Idioma Extranjero -Inglés-e Informática o TIC); y otros en saberes organizados de formas alternativas, a través de talleres (carpintería, cerámica, radio, huerta, ajedrez, etc.). Asimismo varía en el carácter obligatorio u optativo de la extensión de la jornada escolar, la organización de ese tiempo en relación con las edades y niveles escolares; modalidades, tiempos y espacios destinados al trabajo entre docentes, entre otros (Universidad Nacional de Río Negro, 2012).

Ahora bien, el tiempo escolar resulta uno de los elementos que moldean con fuerza los procesos escolares -por eso es considerado uno de los componentes estructurantes o 'duros' de lo escolar- y que está en estrecha vinculación con los restantes aspectos estucturantes de la organización, tales como la transmisión de saberes, la asignación de calificaciones, la división de los alumnos en aulas, la tarea de los docentes (Universidad Nacional de Río negro, 2012).

Ante esto surgen algunas preguntas: ¿Qué vicisitudes encuentran las políticas y programas de extensión de jornada escolar y las instituciones educativas incluidas en estos a la hora de 
URL: http://www.una.ac.cr/educare

intentar modificar la forma clásica de organizar el tiempo escolar y los demás componentes de lo escolar vinculados a aquel? En las instituciones que emprenden este tipo de (re)organización escolar, ¿qué posibilidades de cambio tiene el formato clásico escolar, particularmente, la organización del tiempo y del espacio escolar?

En el apartado que sigue daremos cuenta que el estado actual de la investigación no ha respondido dichas preguntas, por eso repasaremos el estado de la cuestión en los dos ámbitos de conocimientos que, a nuestro entender, convergen en la presente problemática de investigación. Por un lado, los estudios sobre la extensión temporal de la jornada escolar y, por el otro, aquellos realizados en torno a los componentes estructurales y estructurantes de lo escolar. En este último grupo discutiremos las nociones de "gramática escolar" propuesto por Tyack y Cuban (1995) y el concepto foucoultiano de "dispositivo escolar", ahondando en las indagaciones realizadas vinculadas a esta última categoría.

En las conclusiones se proponen preguntas de investigación que surgen, como áreas de vacancia, en el cruce de ambos corpus de investigaciones.

\section{Antecedentes de investigación sobre la extensión de la jornada escolar}

El recorrido realizado al momento por investigaciones vinculadas al tema de la extensión de la jornada escolar permitió encontrar tres grandes líneas de indagación. Por lado, un grupo de investigaciones que vinculan el tiempo que los alumnos pasan en la escuela a variables tales como rendimiento o resultados de aprendizaje o académicos (Belleï, 2009; Belleï, Osses, y Sevilla, 2009; Banco Mundial 2007; Centro de Implementación de Políticas Públicas para la Equidad y el Crecimiento [CIPPEC], 2006; Cotton y Savard, 1981; Department of Education USA, 1994, 1996; Fernández, 2000; Gimeno, 2009; Llach, Adrogué y Gigalia, 2009; Mitter, 1992; Valenzuela, 2002). Por otro lado, investigaciones que dan cuenta de la implementación de diferentes políticas o programas educativos de extensión de la jornada escolar (Department of Education USA 1994, 1996; Fernández, 2000, 2002; Ruz y Madrid, 2005; Zorrilla, Langford, Ramírez y García, 2008). Y finalmente, investigaciones que analizan desde una perspectiva crítica la organización clásica o estándar del tiempo escolar (Aguerrondo, 1998; Escolano, 1992; Fernández, 2000, 2002; Gimeno, 2005; Husti, 1992; Rodríguez, 2009; Varela, 1995).

\section{Investigaciones que vinculan el tiempo que los alumnos pasan en la escuela a variables tales como:rendimiento/resultados de aprendizaje/resultados académicos}

Las investigaciones desarrolladas en los países centrales, desde los estudios clásicos de Cotton y Savard (1981), hasta los más actuales como el de Gimeno (2009), se caracterizan por comparar el comportamiento y vinculación de dichas variables. Son frecuentes los estudios comparativos entre los países de la Organización para la Cooperación y Desarrollo Económico OCDE (Gimeno, 2009) y entre el sistema educativo estadounidense y el japonés. 
Con respecto a los resultados que arrojan esas investigaciones, existe controversia. La mayor parte de los estudios señalan que cuanto mayor es la cantidad de tiempo dedicado, más alto es el nivel de rendimiento de los estudiantes (Cotton y Savard, 1981; Department of Education USA, 1994, 1996; Fernández, 2000, 2002; Mitter, 1992).

Así, por ejemplo, el Reporte de la National Education Commission on Time and Learning de los Estados Unidos (1994) señala:

International comparisons of education are difficult. Cultural factors influence performance and school systems differ. Despite such problems, international comparisons are not impossible and a great deal can be learned from examining schooling abroad. In fact, unflattering comparisons of the academic performance of American students with those from other lands spurred attempts at school improvement in the United States throughout the 1980s. From its review of other nations, the Commission draws several conclusions:

- Students in other post-industrial democracies receive twice as much instruction in core academic areas during high school.

- Schools abroad protect academic time by distinguishing between the "academic day" and the "school day".

- Many of our economic competitors supplement formal education with significant outof-school learning time.

- School performance abroad has consequences and is closely related to opportunities for employment and further education.

- Teachers in other countries enjoy freedom and respect as professional. (Department of Education USA, 1994, Lessons from Abroad, párr. 1-7)

[Es difícil realizar comparaciones internacionales en educación. Los factores culturales influyen en el rendimiento y los sistemas escolares son diferentes. A pesar de estos problemas, las comparaciones internacionales no son imposibles y mucho se puede aprender del análisis de la enseñanza en el extranjero. De hecho, las comparaciones poco halagüeñas sobre el rendimiento académico de los estudiantes americanos comparados con los de otras tierras, ha impulsado los intentos de mejora de la escuela en los Estados Unidos durante la década de 1980. A partir de la revisión de otras naciones, la Comisión establece una serie de conclusiones:

- Los estudiantes en otras democracias postindustriales reciben dos veces más de instrucción en las áreas académicas en la escuela secundaria. 
URL: http://www.una.ac.cr/educare

- Las escuelas en el extranjero protegen el tiempo académico, distinguiendo entre el "día académico" y el "día escolar".

- Muchos de nuestros competidores económicos complementan la educación formal con mucho tiempo de aprendizaje fuera de la escuela.

- El rendimiento escolar de los países extranjeros tiene consecuencias y está estrechamente relacionado con las oportunidades de empleo y educación.

- Los profesores de otros países disfrutan de la libertad y el respeto como profesional". (Departamento de Educación de EE.UU., 1994, Lecciones del Extranjero, Parr. 1-7)]

Por su parte, la última investigación del catedrático español Gimeno (2009), publicada en El valor del tiempo en educación, señala: "el número de horas de clase no está relacionado con los rendimientos de alumnos y alumnas. Finlandia, que es uno de los países que encabezan el ranking en resultados de aprendizajes básicos (según el informe PISA que compara a los países de la OCDE), es el país en el que los alumnos tienen menos horas de clase entre los siete y catorce años" (p. 50). Más adelante agrega:

La literatura especializada en la investigación acerca del tiempo y su relación con los resultados académicos viene a decirnos, en términos generales, que hay poca o ninguna relación entre el tiempo físico ocupado por la escolaridad (calendario y horario) y los resultados académicos comprobables. Escasa y no lineal es la relación entre estos y el tiempo que se ocupa en las tareas académicas". (Gimeno, 2009, p. 66)

Una lectura detenida de las investigaciones que encuentran algún vínculo entre el tiempo escolar y el rendimiento de los alumnos, encuentra que todas estas incorporan ciertos matices a dicha relación. Uno de ellos consiste en discriminar de qué tiempo se trata. El trabajo pionero de Cotton y Savard (1981) sostiene que las investigaciones sobre la relación entre el tiempo escolar y el logro de los estudiantes se centra en tres medidas principales del tiempo de instrucción: a) el tiempo asignado, el cual refiere a la cantidad de tiempo previsto para una actividad de aprendizaje y en el que la oportunidad de aprender está presente; b) el tiempo dedicado a la tarea, que es la cantidad de tiempo en el que se presta atención a una actividad de aprendizaje y tratando de aprender y c) el tiempo de aprendizaje académico, es decir, la cantidad de tiempo empleado por un estudiante en tareas académicas que puede resolver con gran éxito.

De todas las medidas vinculadas al tiempo de aprendizaje de los alumnos, la tasa de tiempo de aprendizaje académico constituye el mejor predictor de éxito. Aclara, asimismo, que mientras que la asignación de tiempo es obviamente necesaria para aprender, aumentar 
el tiempo, sin un aumento comparable en las actividades que facilitan los aprendizajes, es probable que no promueva avances significativos en los resultados. Incluso encuentra que un aumento en el tiempo asignado puede tener una relación negativa con el rendimiento especialmente para los alumnos de alta capacidad.

Mitter (1992) por su parte, afirmará que:

Las comparaciones internacionales, referidas a los rendimientos individuales de los escolares (...) y a la efectividad de la escuela, otorgan a la duración de la formación escolar una gran importancia como magnitud de medida. La cuestión central es (...) hasta qué punto dependen dichos rendimientos (...) del tiempo que los alumnos pasan en el centro docente y, de otro, cómo se distribuye dicho tiempo. (p. 221)

También en Latinoamérica se han identificado estudios que han analizado esta relación. En general refieren los resultados de la implementación de ciertas políticas, como es el caso del estudio de Llach et al. (2009), referido a la ciudad de Buenos Aires (Argentina) y de los realizados por el Ministerio de Educación de Chile, (2003), la Universidad Católica de Chile (Ruz y Madrid, 2005) y por Valenzuela et al. (2009) sobre la política de ampliación de jornada desarrollada en ese país en los 90, o de programas de extensión de jornada, tal es el caso de los estudios del Banco Mundial (2007) y la Administración Nacional de Educación Pública de Uruguay sobre la implementación del Programa de Escuelas Completas en ese país (Administración Nacional de Educación [ANEP], 2003). En el caso de Chile, cabe citar los estudios de Belleï (2009) y Valenzuela et al. (2009).

Valenzuela et al. (2009), por ejemplo, al identificar las causas que explican el mejoramiento de los resultados obtenidos por los estudiantes chilenos en PISA 2006 respecto a PISA 2001 sostiene:

La aplicación de la Jornada Escolar Completa ha significado un aumento promedio de resultados, especialmente para los alumnos de rendimientos medio y bajo, todo lo cual es una buena noticia; sin embargo, estos aumentos han sido pequeños para el volumen de recursos involucrados, lo cual sugiere un uso poco eficiente del tiempo escolar adicional, en efecto, el estudio mostró que no ha habido progresos en la efectividad de la JEC. (Valenzuela et al. 2009, p. 85)

En el caso de Uruguay, la implementación de la Escuelas de Tiempo Completo es analizada por el Informe No 38082-UY del Banco Mundial (del 9 de marzo de 2007). Dicho informe, pondera factores que influyen en la calidad y la equidad del sistema de educación de Uruguay, con el 
URL: http://www.una.ac.cr/educare

objetivo de identificar sus prioridades. Además, el mismo presenta evidencias del impacto positivo del programa de Escuelas a Tiempo Completo en el aprendizaje. El programa parece ser efectivo, especialmente en las escuelas que sirven segmentos de la población de contexto socioeconómico más desfavorable.

El informe señala:

El programa mejoró de manera significativa el rendimiento de los alumnos en las escuelas que participaron, especialmente en matemáticas [sic]. Es interesante notar que el impacto es mayor cuando se restringe la muestra analítica solo a aquellas escuelas que atendían a la población más desfavorecida y que tenía la peor infraestructura antes de convertirse en escuelas de tiempo completo. Los autores calculan que en estas escuelas hubo un aumento de las calificaciones de 0,30 puntos en Matemáticas y de 0,20 puntos en Lenguaje por cada año de participación en el programa. Por lo tanto, un ciclo de seis años de educación primaria resultaría en un aumento de 1,8 puntos en matemáticas [sic] y de 1,2 puntos (de 24) en lenguaje [sic]. En estas escuelas, donde la calificación promedio es de 12 y donde casi 60 por ciento de los alumnos repite al menos un curso en primaria, un aumento de casi dos puntos aumentaría el número de estudiantes que aprueban el grado entre 10 y 15 por ciento. Este impacto es significativo, especialmente si se considera la población que se beneficia de estas escuelas. (Banco Mundial 2007, p. 36)

La ANEP (2003) del Uruguay estudió los resultados de las escuelas de tiempo completo en su Segundo Informe sobre los Resultados de la Evaluación Nacional de Aprendizajes en Lenguaje y Matemática, aplicada en alumnos de $6^{\circ}$ año de educación primaria en 2002. Trabajando con estadísticas descriptivas y agrupando a los alumnos en quintiles, en función del contexto sociocultural de sus familias, el informe encuentra que las escuelas de tiempo completo presentan mejores resultados que aquellas de jornada simple para los primeros dos quintiles (denominados en el informe como de contexto sociocultural "muy desfavorable" y "desfavorable"). En el caso de la evaluación de lenguaje un 3.6\% más de alumnos alcanzan el nivel de suficiencia (60\% de las respuestas correctas) en el contexto muy desfavorable y un $4.9 \%$ más en el contexto desfavorable, mientras que para Matemática estos porcentajes son de $7.5 \%$ y $6.8 \%$ respectivamente. Por el contrario, en el tercer quintil (de contexto sociocultural "medio") los resultados en ambas disciplinas son menores para las escuelas de tiempo completo, $4.6 \%$ en Lenguaje y $7.6 \%$ en Matemática. Cabe aclarar que solo las diferencias para Matemática resultaron estadísticamente significativas.

En el caso de Argentina, no se dispone de trabajos específicos que hayan analizado esta temática. El único trabajo identificado es el desarrollado por el CIPPEC en el año 2006, el cual, con el fin de realizar una primera aproximación a esta relación, analiza los resultados de las 
pruebas de los Operativos Nacionales de Evaluación, aplicados de manera censal en el $6^{\circ}$ año de la EGB en el año 2000. De dicho análisis surge que:

(...) los alumnos de establecimientos con JC obtienen en general mejores rendimientos que los alumnos de jornada simple, aunque la diferencia es en todos los casos leve. La mayor diferencia se observa en el tercil de más alto nivel socioeconómico, resultado sin dudas llamativo y que deberá ser explorado a fin de identificar los posibles determinantes de esta situación. (CIPPEC, 2006, p. 24)

En estas investigaciones también se estudian otras dimensiones asociadas: en mayor medida encontramos investigaciones sobre el impacto financiero para los sistemas educativos de la extensión de la jornada escolar (Banco Mundial, 2007; CIPPEC, 20063; Mitter, 1992;) y en menor medida hay estudios sobre el impacto de tal ampliación en la fatiga de los alumnos o la relación entre tiempo escolar y tiempo extraescolar (Gimeno, 2009).

Un análisis de estos trabajos nos permite observar que, en general, refieren al tiempo escolar objetivo pre-definido, que se mide, se ordena, se distribuye en horarios, jornadas, ritmos, edades, secuencias, etc. Asimismo, un común denominador en los resultados de las investigaciones parece ser que el vínculo entre tiempo escolar y rendimiento de los alumnos es poco significativo; fundamentalmente en el caso de alumnos pertenecientes a sectores sociales medios y al compararlo con los recursos comprometidos en dichas experiencias.

Si bien los estudios no avanzan en explicar las razones de dicho fenómeno, varios advierten (CIPPEC, 2006; Gimeno, 2009; Mitter, 1992) que "lo estrictamente importante en el tratamiento del tiempo que se hace en educación no es su duración (...) sino lo que en él se hace" (Gimeno, 2009, p. 50). Lo que otros definirán como 'calidad del tiempo' (Department of Education USA, 1996). O en palabras de Mitter (1992) (...) habría que conceder mayor peso a la cuestión bastante descuidada (...) de las posibles alternativas a la estructuración actual del tiempo escolar, de forma que éste se ajuste mejor a la capacidad de rendimiento y a las necesidades de aprendizaje de cada escolar de lo que la reglamentación vigente en el sistema educativo ha permitido hasta ahora (p. 233).

Ahora bien, en este punto de la discusión algunas interrogaciones quedan sin respuesta: ¿por qué más tiempo del tradicional no genera los efectos positivos que se esperan en los resultados educativos de los alumnos? ¿Qué supone, en términos de organización escolar, más tiempo; pero de otra calidad?

3 Dicho estudio concluye: “En total, si se asume que la política alcanzará la meta más modesta del 20\% de la matrícula de la EGB completa, los costos para el primer año alcanzarían los $\$ 1.748$ millones y en el año 2010 ascenderían a $\$ 3.070$ millones, aunque a partir de 2011 disminuirían a $\$ 1.762$ (dado que finalizaría la etapa de construcción de escuelas). Esto representaría un 0,29\% del PBI en 2007 y un 0,46\% en 2010 , o un $5,7 \%$ del gasto educativo total consolidado en 2007 y un 7,6\% en 2010". (CIPPEC, 2006, p. 58) 


\section{Investigaciones que dan cuenta de los procesos de implementación de la extensión de la jornada escolar}

Cabe señalar los estudios del Department of Education USA (1994, 1996). La National Education Commission on Time and Learning dirigida por el gobierno federal, preocupado por el rendimiento estudiantil en los Estados Unidos, se propuso como objetivo llevar a cabo un examen exhaustivo de ciertas reformas desarrolladas en algunos estados americanos, en relación con el tiempo escolar.

Sobre la base de su investigación, la Comisión definió las dimensiones del desafío que enfrentan las escuelas de EE.UU referidas al tiempo, sosteniendo que el reloj y el calendario fijo es un defecto de diseño fundamental que debe ser cambiado; que la jornada escolar debe ser modificada para responder a los grandes cambios que han transformado la vida estadounidense fuera de la escuela; que los educadores no tienen el tiempo que necesitan para hacer su trabajo correctamente, y que alcanzar los estándares de clase mundial requiere más tiempo para casi todos los estudiantes (Department of Education USA, 1994).

En el informe The Uses of Time for Teaching and Learning, publicado en octubre de 1996, se establecen tres áreas muy amplias donde se debe investigar: la cantidad de tiempo en la escuela (estudios sobre el impacto en los resultados educativos de la ampliación del año escolar y de la jornada escolar), la calidad del tiempo en la escuela, y el uso del tiempo fuera de la escuela para complementar el aprendizaje escolar. Sobre la calidad del tiempo en la escuela hacen alusión a la necesidad de investigar lo que denominan variables fundamentales respecto a la calidad de las cuestiones del tiempo: currículo, enseñanza, evaluación y estructuras de la organización escolar. Asimismo sugieren investigar la naturaleza del tiempo de los maestros en un contexto profesional, la organización de nongradedness o agrupación de distintas edades y las nuevas alternativas para la programación de la cantidad tradicional de tiempo escolar (Department of Education USA, 1996).

Es decir, lo que este estudio muestra es cómo la calidad del tiempo escolar está dada por el vínculo de dicha variable con otros componentes de lo escolar: lo curricular, la enseñanza, la evaluación, los agrupamientos, etc.

También es pertinente resaltar los estudios de Fernández $(2000,2002)$ en los que analiza la implementación, en varias jurisdicciones españolas, del cambio de jornada partida a jornada continua. A partir del análisis de los proyectos y solicitudes de cientos de centros educativos, de las entrevistas realizadas a medio centenar de informantes privilegiados y protagonistas significativos del proceso (sindicalistas, representantes de los padres, autoridades, expertos) y de la visita a una veintena de colegios, fundamentalmente en Canarias, Andalucía, Toledo y Alcalá de Henares, el autor señala que el cambio, promovido por los docentes, favorece a las clases medias funcionales (profesionales, técnicos, funcionarios, oficinistas) que dan gran importancia a complementar el programa de estudios básicos con un conjunto de saberes y capacidades complementarias (lenguas extranjeras, informática, música, actividades deportivas más sofisticadas) y al mismo profesorado. La víctima de este proceso son los sectores sociales en 
los que se concentran mayores déficits económicos y culturales en relación con lo que la escuela requiere. Señala, asimismo, que la evidencia disponible sobre rendimiento, fatiga, atención, etc. en el aula, si bien es poca, es contraria a la concentración horaria. Tampoco es conclusivo en relación con el rendimiento.

La Evaluación del Diseño del Programa Nacional de Horario Extendido en Primaria, Programa Nacional Escuelas de Tiempo de México, desarrollado a solicitud del mismo Programa por Zorrilla et al., de la Universidad Autónoma de Aguascalientes en el año 2008, es también importante de destacar. Dicha evaluación se realizó mediante análisis de las fortalezas, oportunidades, debilidades y amenazas del diseño. Si bien coinciden en que la ampliación del tiempo de la jornada escolar es una estrategia válida para incrementar las oportunidades de aprendizaje sobre todo en los niños que proceden de ambientes de mayor vulnerabilidad, los autores advierten, entre otras cuestiones, que la propuesta del programa no cuenta con las orientaciones pedagógicas y de gestión que exige una concepción basada en incrementar las oportunidades de aprendizaje ni con una estrategia fincada en la extensión del horario de la jornada escolar diaria. "(...) La sola permanencia de los alumnos dentro de la escuela en un horario extendido, no garantiza per se, ni el incremento de las oportunidades de aprendizaje y por ende tampoco el logro de una educación integral" (Zorrilla et al., 2008, p. 42).

También es interesante subrayar el estudio realizado por la Dirección de Estudios Sociológicos de la Universidad Católica de Chile (DESUC), por encargo del Ministerio de Educación, en el año 2005. Se trata de un investigación de representatividad nacional, basada en la extracción de muestras probabilísticas de establecimientos, docentes, padres y apoderados y alumnos. La misma analiza, por un lado el nivel de cumplimiento a las normas en lo referente al tiempo global de la jornada y al cumplimiento del tiempo pedagógico (Plan de estudio + libre disposición). El estudio señala al respecto:

(...) El promedio de tiempo destinado a almuerzo se ubica por sobre lo esperado normativamente, y a la inversa, el tiempo promedio destinado a recreos, se ubica bajo la referencia normativa. (...) Cerca de la mitad de las escuelas en JEC (47,8\%), están destinando un tiempo pedagógico total muy cercano al esperado según la normativa. Otro porcentaje no menor (26,9\%) está destinando tiempos pedagógicos por sobre la norma fijada. En contraste, existe un grupo de escuelas $(25,3 \%)$ que se encuentran bajo el tiempo pedagógico esperado. (...) Si se observa específicamente el cumplimiento de horas del Plan de Estudios, se constata que, el 83, 2\% de las escuelas está en o por sobre el tiempo de dedicación semanal previsto. Sólo un 6,7\% de las escuelas muestra tiempos semanales destinados al Plan de Estudios bajo las 28 horas semanales. (...) En el caso de las horas de libre disposición, se constata que sólo un $17,1 \%$ de los establecimientos destina más de las 8 horas semanales previstas por norma a este concepto. Más frecuentemente $(47,8 \%$ de los casos) las escuelas, se mantienen en el rango de 6 a 8 horas semanales dispuestas para 
URL: http://www.una.ac.cr/educare

actividades de libre disposición. Cerca de un tercio de escuelas $(35,1 \%)$, destinan menos de 6 horas semanales a este tipo de actividades. (DESUC, 2005, pp. 13, 15 y 18)

Se señala que el grado de cumplimiento es alto y se agrega que en las horas de 'libre disposición' las principales actividades realizadas por los establecimientos son las artísticas (talleres de manualidades-artesanía, taller de música, y artes plásticas), deportivas y tecnología y computación, donde la más común es el taller de computación.

Por otro lado, el estudio indaga las percepciones y evaluaciones de los actores, el significado de la jornada escolar completa (JEC) para docentes, padres y alumnos. En tal sentido se estudió el nivel de participación en la formulación del proyecto; la prioridad dada a distintos factores para la implementación de la JEC; la evaluación de funcionamiento de la jornada escolar completa; sus efectos desde la percepción de los actores; en relación a la familia: el nivel de acuerdo con horarios de entrada-salida de los alumnos y razones desacuerdo; la percepción de necesidad de tiempo para realizar tareas escolares e influencia de la JEC en dicha necesidad; las actividades a las que los alumnos destinan su tiempo libre; el rol de la familia en la educación de los hijos; la influencia de la JEC en la economía familiar; las expectativas de los padres respecto al futuro educacional de sus hijos; el medio y tiempo de traslado al colegio utilizado por los alumnos; el nivel de motivación de los alumnos y rendimiento; la situación laboral de actores del establecimiento.

Asimismo, el estudio identifica que de acuerdo con la percepción de los actores, la jornada completa ha tenido efecto sobre el desarrollo de conocimientos y habilidades de los alumnos: fundamentalmente en mejorar la formación en valores, deportes y nuevas tecnologías; en el aprovechamiento de la infraestructura, equipamiento y recursos de los edificios escolares, en la calidad del trabajo en equipo y las prácticas pedagógicas docentes y en la disminución del tiempo en que los niños están solos en su casa o en la calle; en el aumento de posibilidades para que los padres o tutores trabajen, etc.

No se constatan percepciones relevantes que imputen como efecto de la JEC un mejoramiento específico en las áreas de aprendizaje tales como lenguaje, matemáticas y ciencia. (...) efectos específicos de la JEC sobre la economía familiar, según la percepción de los padres, destaca la posibilidad de que la madre pueda trabajar remuneradamente (44\% de menciones), el ahorro del costo de almuerzo de sus hijos (37\%) y la posibilidad de evitar el costo de dejar el cuidado de los niños con terceros (35,7\%). (DESUC, 2005, p. 136)

Finalmente, citaremos el estudio desarrollado por el CIPPEC (2006) sobre las políticas de extensión de la jornada escolar en Argentina. Dicha investigación señala que las escuelas de 
jornada completa y de jornada extendida, creadas en distintas provincias argentinas en los últimos veinte años, han tenido la finalidad de incrementar la justicia social, por lo que se ha focalizado en escuelas consideradas de alta vulnerabilidad social.

El Informe del CIPPEC (2006) señala, sin embargo, disparidad de criterios para incluir las escuelas en dichos programas, a saber: la disponibilidad de infraestructura edilicia, los indicadores de fracaso escolar y el requisito de presentar proyectos institucionales por parte de las escuelas. El estudio agrega que dichas políticas explicitan los contenidos a abordar en el tiempo extra, con prioridad para cuatro tipos de saberes:

Los contendidos "básicos" o "prioritarios" (lengua y matemática en primer lugar, y ciencias naturales y sociales en segundo). (...) Áreas "curriculares" y disciplinas ligadas con las "necesidades actuales" (educación física, música, artística así como idiomas e informática o tecnología). (...) Saberes organizados de formas alternativas, por ejemplo, a través de talleres (carpintería, cerámica, radio, huerta, ajedrez, etc.). (CIPPEC, 2006, p. 43)

Como puede observarse, estas investigaciones dan cuenta de distintos aspectos de los procesos de implementación de la extensión de la jornada escolar, además, en diferentes localizaciones: algunos enfatizan en la formulación de los programas (Zorrilla et al., 2008), otros en el nivel de cumplimiento de las pautas normativas y el nivel de satisfacción de los actores involucrados (DESUC, 2005); otros en las diferentes modalidades de implementación (CIPPEC, 2006; Department of Education USA, 1994, 1996; Fernández, 2000, 2002).

También se observa que, si bien se trata de programas o políticas diferentes -a excepción del caso español del pasaje de la jornada continua a la jornada partida (Fernández, 2000, 2002)-, todos coinciden en que estas reformas tienen el objetivo de crear condiciones institucionales que mejoren cuantitativa y cualitativamente los aprendizajes de los alumnos destinatarios.

Resulta interesante en esta indagación que algunos de los estudios concluyan que para cumplir ese objetivo, el diseño estándar debe ser modificado (Department of Education USA, 1994) o que un cambio cualitativo en el tiempo escolar implica redefinir otros componentes de lo escolar: lo curricular, la enseñanza, la evaluación, los agrupamientos, etc. (Department of Education USA, 1996).

Ahora bien, ninguna de estas investigaciones dan cuenta en qué consisten esas condiciones estándares y cómo las mismas se (re)organizan a partir de la ampliación de la jornada. Tampoco indagan qué vicisitudes encuentran las políticas y programas de extensión de jornada escolar y las instituciones educativas incluidas en estos a la hora de intentar modificar la forma clásica de organizar el tiempo escolar y los demás componentes concurrentes; en las instituciones que emprenden este tipo de (re)organización escolar qué posibilidades de cambio tiene el formato escolar clásico. 


\section{Investigaciones que analizan, desde una perspectiva crítica la organización clásica o estándar del tiempo escolar}

Mientras varias de las investigaciones ya analizadas, (Departamento de Educación EEUU, 1994, 1996; Ruz y Madrid, 2005, Zorrilla et al., 2008) conciben el tiempo escolar solo en su dimensión objetiva y monocrónica, es decir, un tiempo pre-definido, que se mide, se ordena, se distribuye (horarios, jornadas, ritmos, edades, secuencias), muchas veces considerándose equivalente el tiempo empleado y el aprendizaje realizado. Existe otra línea de investigaciones (Aguerrondo, 1998; Escolano, 1992; Fernández, 2000, 2002; Gimeno, 2009; Husti, 1992; Rodríguez, 2009; Varela, 1995) que analiza críticamente la forma de organización escolar del tiempo e incluso sugiere otras, como es el estudio de Husti (1992).

Estos autores coinciden en que la jornada escolar mantiene su estructura rígida originaria del siglo XIX, basada en una concepción técnico-racional, según la cual las unidades de tiempo son uniformes y pre-definidas:

(...) este orden se estructura a partir del módulo de la hora; tiempo que sirve tanto para la clase de lengua como la de matemáticas y que se aplica en todos los niveles. Las jornadas se organizan en unidades horarias y la suma de aquéllas origina la semana. El año o curso es el resultado acumulativo de un número invariable de semanas, sólo interrumpido por los períodos de asueto y vacación. Tal construcción del tiempo escolar responde al paradigma mecanicista newtoniano (...). (Escolano, 1992, p. 60)

Los resultados de esas investigaciones afirman que ese orden entra en contradicción y conflicto con los cambios acontecidos en los dos últimos siglos tanto en el ámbito social como en el escolar (Escolano, 1992; Fernández, 2000, 2002; Husti, 1992). En tal sentido Escolano (1992) señala que muchos aspectos de la enseñanza han cambiado (programas, métodos y exámenes, entre otros), pero el tiempo escolar y su utilización se han mantenido resistiendo a todo tipo de reforma.

A lo que Husti agrega:

La institución escolar está en completa contradicción consigo misma al imponer el modelo de organización secular, inmutable y uniforme, y desear al mismo tiempo cambios profundos, fijándose para ello una serie de objetivos -como, por ejemplo, abrir la escuela a su entorno, utilizar la tecnología moderna, tener en cuenta la heterogeneidad de los alumnos, diversificar las prácticas pedagógicas y las fuentes de conocimiento y, de forma particular, fomentar la participación activa del alumno en la construcción de sus conocimientos; objetivos, todos ellos, que requieren una planificación del tiempo variable y adaptable, es decir, móvil. (Husti, 1992, p. 273) 
Los autores que revisan críticamente la organización temporal de lo escolar recuperan a la hora del análisis otras dimensiones del tiempo escolar, tales como la dimensión personal y subjetiva que remite a los contenidos y sentidos que el tiempo escolar tiene para los sujetos (Gimeno, 2005); la dimensión socio cultural, considerando que el tiempo de la educación cumple las funciones de estructurar las relaciones sociales, expresar y servir para establecer diferencias y jerarquías dentro de las mismas entre grupos e individuos (Gimeno, 2005) a la vez que, como señala Escolano (1992), establece las primeras pautas reguladoras del tiempo vivido en la infancia, en la estructuración de la vida humana. Afirma:

(...) sus códigos no son sólo un sistema de la escuela, sino las claves de la cronobiología y la cronocultura, las señales que organizan las primeras percepciones cognitivas de la temporalidad (una de las categorías estructurales de los individuos y las sociedades) (...). (Escolano, 1992, p. 57)

Este último autor, en su estudio histórico sobre la configuración y cambios en el calendario escolar, concluye que:

(...) el orden del tiempo escolar es, además de un sistema de cómputo y de planificación, una fórmula de poder, un compromiso entre la política y las costumbres, entre los intereses de la economía y de los profesionales de la enseñanza y la racionalidad organizativa de la escuela, entre los criterios de modernización y la liturgia. (Escolano, 1992, p. 77)

Esta línea conceptualiza el tiempo escolar como principal condicionante de los procesos escolares (Fernández, 2002). Así, Aguerrondo (1998) lo ubica como una de las condiciones básicas institucionales (CBI) que tienen que ver con las decisiones que se toman en relación con qué, cómo, cuándo y dónde enseñar. Ella afirma:

(...) aspectos que moldean con más fuerza la compleja trama de procesos que se desarrollan en la escuela -por esto se los denomina aspectos estructurantes- y conforman el campo organizacional de la institución escuela. Estos aspectos son el tiempo, el espacio, los agrupamientos y la presencialidad (...). La modificación de la planificación del tiempo escolar está en estrecha vinculación con los restantes aspectos estructurantes de la organización, tales como el espacio, los agrupamientos, la presencialidad". (Aguerrondo, 1998, p. 21) 
URL: http://www.una.ac.cr/educare

Varela (1995), por su parte, analiza la forma en que las "categorías espacio -temporales, saberes, poderes, pedagogías y formas de subjetivación constituyen dimensiones que se cruzan, se imbrican y se ramifican en el interior de las instituciones educativas" (Varela, 1995, p. 159) y establece que cada tipo de pedagogía conlleva una particular definición témpore espacial.

Ahora bien, ninguna de estas indagaciones que analizan desde una perspectiva crítica la organización clásica o estándar del tiempo escolar han reparado en las políticas, programas o experiencias educativas que desde hace más de dos décadas toman como variable de cambio, precisamente, la organización temporal. ¿No son, acaso, estas políticas oportunidades regias para analizar el comportamiento de la organización escolar ante los intentos de modificar uno de sus componentes duros, como lo es el tiempo? Estas políticas resultarían laboratorios naturales para analizar cuánto resiste la organización clásica del tiempo escolar, en qué cambia y cómo esos cambios (de ocurrir) afectan los restantes componentes estructurantes de lo escolar (espacios, agrupamientos, etc.).

\section{Antecedentes de investigación sobre componentes estructurales y estructurantes de lo escolar: la gramática escolar y el dispositivo escolar}

Los componentes estructurales y estructurantes de lo escolar, es decir, aquellos elementos invariantes que constituyen y dan forma a lo escolar, han sido transformados en objetos de indagación por parte de diferentes disciplinas y perspectivas teóricas. Numerosos autores (Baquero, Diker, y Frigerio 2007; Baquero y Terigi, 1996; Bosco, 2000; Dicker, 2005; Dussel, 2006; Gvirtz y Larrondo, 2007; Hargreaves y Goodson, 2006; Julia, 2001; Tyack y Cuban, 1995; Viñao, 2002) han reparado en aquellos elementos invariantes que organizan la experiencia escolar a la hora de explicar aquello que en lo escolar permanece casi inalterado, a pesar de los intentos reformadores y que explican el fracaso de tales reformas ${ }^{4}$; o el comportamiento de lo escolar ante la inclusión de la novedad ${ }^{5}$, las crisis del modelo o forma escolar hegemónica ante las modificaciones sociohistóricas y en la subjetividad acaecidas en más de dos siglos.

Al revisar las investigaciones arriba citadas, es posible identificar, por lo menos, dos grandes tradiciones a la hora de conceptualizar esos elementos invariantes, la 'caja negra' de lo escolar, tal lo ha caracterizado Julia (2001). Una de esas tradiciones recupera la categoría de 'gramática escolar' propuesta por los historiadores estadounidenses Tyack y Cuban (1995) y la otra se afilia a la conceptualización foucoultiana de 'dispositivo'.

La categoría de gramática escolar surge de un estudio pormenorizado de las reformas educativas desarrolladas en los últimos cien años en los Estados Unidos. Es definida como las

${ }^{4} \quad$ Aquí podemos citar el trabajo de Dussel sobre gestión y desarrollo curricular en países de América Latina y el de Romero (2006) sobre cambio educativo y asesoramiento escolar.

5 Celulares y computadoras (Gvirtz y Larrondo, 2007), medios de comunicación electrónico (Sapoznikow, 2008). 
"estructuras, reglas y prácticas que organizan la labor de la instrucción" e "incluyen prácticas tan familiares como la graduación de los alumnos por edades, la división del conocimiento por materias separadas y el aula autónoma con un solo maestro" (Tyack y Cuban, 1995, p. 23).

Si bien este concepto es ampliamente desarrollado en un texto hace tiempo agotado en la Argentina, es importante señalar algunas de sus notas distintivas. La gramática escolar "es un producto de la historia y no una creación primordial" (Tyack y Cuban, 1995, p. 169); como sucede con la gramática de la lengua, su estructura y reglas no necesitan ser comprendidas a nivel de la conciencia para operar correctamente; regula la conducta y el trabajo de quienes habitan la escuela y define lo que, para la mayoría de los actores sociales, es 'una verdadera escuela'6.

Para Tyack y Cuban (1995), como se cita en Romero (2006), "(...) lo que ha permitido que se mantenga la gramática de la escuela no es tanto un conservadurismo consciente, como la falta de reflexión sobre los hábitos institucionales y las creencias culturales ampliamente extendidas sobre lo que constituye una 'escuela real' "' (p. 47).

Esta categoría ha resultado particularmente fértil a la hora de explicar el comportamiento de la escuela ante reformas gestadas en los niveles centrales, como es el caso de los estudios sobre reformas en el sistema educativo norteamericano de Hargreaves y Goodson (2006), o los estudios locales de Dussel (2006) y Romero (2006); o ante la incorporación de los medios de comunicación electrónicos (Sapoznikow, 2008); las nuevas tecnologías de la información (Bosco, 2000), el celular y la computadora (Gvirtz y Larrondo, 2007).

Asimismo existe una larga tradición que piensa la escuela en clave foucoultiana. Desde la pionera obra Trabajos elementales sobre la escuela primaria, de Querrien (1994), hasta la compilación de trabajos generados en la década de los 80 por Varela y Álvarez Uría (1991) y presentados en Arqueología de la escuela, al conceptualizar la escuela recuperan la categoría de "dispositivo" (Foucault, 1977).

Otros investigadores que optan por la "caja de herramientas" foucoultiana para analizar el campo de la educación son:

Larrosa (1995) quien sugiere una perspectiva teórica, en clave foucoultiana, para el análisis de las prácticas pedagógicas que construyen y median la relación del sujeto consigo mismo; esa relación en la que se establece, se regula y se modifica la experiencia que uno tiene de sí mismo, la experiencia de sí. (Larrosa, J., 1995, pp. 262, 263).

Foucault nunca dedicó a la educación un trabajo sistemático y acabado, pero se refiere a pedagogías, sistemas educativos, dispositivos de examen, etc., en sus libros y entrevistas. Es en Vigilar y castigar (Foucault, 1997) donde realiza las alusiones más explícitas a la educación, fundamentalmente al peso que tuvieron las instituciones educativas en el desarrollo de las tecnologías disciplinarias. En esos trabajos termina de formular su concepto de dispositivo. Agamben (2005) planteará que

6 Sedimentada a lo largo de décadas, esta gramática habría sido interpretada por alumnos y profesores como los rasgos constitutivos de una escuela "verdadera" o "auténtica". 
«dispositivo» "es un término técnico decisivo en la estrategia del pensamiento de Foucault. Lo usa a menudo, sobre todo a partir de la mitad de los años setenta, cuando empieza a ocuparse de lo que llamó la «gubernamentalidad» o el «gobierno» de los hombres.

En tal sentido no es difícil coincidir con Dussel (2006) cuando afirma que "la noción de dispositivo se ha usado un tanto abusivamente, (...) ha sido utilizada para hablar de cualquier conjunto o tecnología; así, los cuadernos de clase, la arquitectura o la formación docente son considerados dispositivos" (Dussel, 2006, p. 90).

Pero más allá del uso más o menos fiel a la propuesta focoultiana, nos parece interesante proponer una lectura de la escuela primaria como dispositivo, en tanto ayuda a pensarla en su combinación particular de elementos, de reglas y normas, en su configuración simultánea de saberes, sujetos y tecnologías.

Con tal permiso teórico, este concepto $-y$ otros vinculados al mismo como el de "ensambles de tecnologías o maquinaria" (Bosco, 2000)-, ancló creativamente en la intelectualidad educativa argentina y ha resultado sumamente fértil para analizar las configuraciones que ha adoptado y adopta "lo escolar», sus variaciones actuales, sus puntos de tensión, sus desbordes.

En los numerosos trabajos que se inscriben en esa línea de pensamiento se analizan la construcción histórica del modelo de la escuela primaria común argentina, sus rasgos distintivos y las tecnologías priorizadas ${ }^{7}$ (Baquero, Dicker, 2005; Diker, y Frigerio, 2007; Dussel, 2006; Gvirtz y Larrondo, 2007; Terigi, 2006) y lo que esas formas estandarizadas generan: tipos de aprendizajes, representaciones acerca de qué es aprender, tipos subjetivos (docentealumno) y fenómenos como la violencia (Baquero, 2001; Baquero y Terigi, 1996; Corea, 2000) y experiencias y situaciones en las que algo de las formas estandarizadas de ordenar la escolaridad son modificadas: propuestas de no gradualidad, los grados de aceleración, el plurigrado de las escuelas rurales (Terigi, 2002, 2006, 2007a), la incorporación de las nuevas tecnologías y de la llamada cultura mediática (Bermúdez, s. f.), estudios de casos de escuelas que implementan experiencias alternativas de escolaridad (como las escuelas de familia agrícola y escuelas de reingreso investigadas por Southwell, 2009).

A continuación se profundizará el desarrollo de estos trabajos y lo que ellos nos aportan para pensar la organización escolar, en términos de dispositivo.

\section{Michel Foucault y más allá: pensar el dispositivo escolar y sus posibilidades de reorganización}

Un trabajo pionero de sociología de la educación que adopta la perspectiva foucoultiana es la obra de Querrien (1994), Trabajos elementales sobre la escuela primaria, que fuera editado

\footnotetext{
Papel, libros, pizarrones, cuadernos, guardapolvos, actos escolares, entre otras, fueran las tecnologías utilizadas en el siglo XIX pero también en la mayor parte del siglo XX. Dussel señala que "estas tecnologías adquieren una historia propia, una forma de configurarse que perdura en el tiempo y que se convierten en parte del núcleo duro de lo que se entiende por escuela" (Dussel, 2006, p. 98).
} 
por primera vez por La Piqueta en 1979. En este, a partir de una pesquisa genealógica sobre la escuela primaria francesa y sus transformaciones a partir del siglo XVII, se dará cuenta de la génesis y consolidación de algunos elementos del dispositivo escolar, lo que resulta particularmente significativo para esta indagación.

Querrien (1994) identificará los siguientes elementos del dispositivo escolar: la obligatoriedad, el carácter simultáneo de la enseñanza, la simplificación y unificación del espacio y el tiempo escolar, el lugar hegemónico de la mirada en la escuela y el sometimiento del cuerpo.

Lo propio hace Varela (1991, 1994 y 1995) para el caso español, quien señala que lo que caracteriza a la escuela es fundamentalmente "la definición de un estatuto de la infancia, la emergencia de un espacio específico destinado a la educación de los niños, la aparición de un cuerpo de especialistas de la infancia dotados de tecnologías específicas y de 'elaborados' códigos teóricos; la destrucción de otros modos de educación y la institucionalización propiamente dicha de la escuela: la imposición de la obligatoriedad escolar decretada por los poderes públicos y sancionada en leyes (Varela, 1991, p. 15).

Varela (1995) también analizara la forma en que "categorías espacio-temporales, saberes, poderes, pedagogías y formas de subjetivación constituyen dimensiones que se cruzan, se imbrican y se ramifican en el interior de las instituciones educativas" (Varela, 1995, p. 159) y lo ilustrará con la definición de tres tipos ideales pedagógicos que se corresponden con tres períodos históricos distintos, a saber: las pedagogías disciplinarias, las correctivas y las psicológicas.

Como adelantamos más arriba, el rastreo bibliográfico realizado también nos ha permitido identificar, en el campo de la investigación educativa argentina, un número considerable de trabajos que se ocupan de los elementos invariantes de lo escolar, de aquello que le otorga identidad. La mayoría de ellos aluden al término dispositivo, aunque su utilización, como nos advierte Dussell (2006), ha sido un tanto abusiva.

Más allá del uso más o menos fiel a la propuesta focoultiana, los trabajos que pasaremos a detallar poseen la fertilidad de dar cuenta de la combinación particular de elementos, la configuración simultánea de saberes, sujetos y tecnologías que hacen a la escuela primaria argentina. Es decir, recuperamos estas investigaciones por su potencia para describir elementos del dispositivo, más allá de su filiación teórica.

Un grupo de trabajos da cuenta de la conformación histórica de lo que podríamos denominar "escuela primaria argentina" (Baquero, Diker y Frigerio, 2007, Dicker, 2005; Dussel, 2006; Gvirtz y Larrondo, 2007; Pineau, 2001; Terigi, 2006); otro grupo ha reparado en experiencias y situaciones en las que el dispositivo se modifica (Southwell, 2009 y Terigi, 2002, 2006, 2007a, 2007b) y un tercer grupo repara en el papel del dispositivo escolar en los procesos de subjetivación (Baquero, 2001; Baquero y Terigi, 1996; Corea, 2000). 
Pineau (2001) se preguntará qué es la escuela y analizará cuáles fueron los desarrollos y elementos que permitieron que esta, en tanto forma educativa institucionalizada, se impusiera en todo el globo hacia fines del siglo XIX. El autor punteará "(...) algunas de las piezas que se fueron ensamblando en una extraña amalgama no exenta de contradicciones, para dar lugar al fenómeno escuela, y sobre las que se apoyan las rupturas y reordenamientos que produce al interior del campo pedagógico" (Pineau, s. f., p. 3).

Algunos de estos elementos se inspiran, claramente, en los trabajos de Querrien (1994) y Varela $(1991,1994)$ ya citados, a saber: la aparición del espacio cerrado como dispositivo institucional y la ubicación de un espacio y tiempo específicos, la destrucción de otras formas educativas; la formación de un cuerpo de especialistas dotados de tecnologías específicas y la definición de un estatuto específico de la infancia.

Pineau (2001) identificará otros componentes del dispositivo, como la construcción de una realidad colectiva, pues la escuela se presenta como una forma de enseñar a muchos a la vez; su pertenencia a un sistema mayor; el establecimiento de una relación asimétrica entre maestro y alumno; la generación de artefactos específicos de control de los «cuerpos dóciles»; el establecimiento de un sistema de trasmisión de saberes íntimamente vinculado al funcionamiento disciplinario; la determinación de los "saberes elementales", el desarrollo de prácticas y currículos altamente uniformes, la predeterminación y sistematización de los contenidos; la instauración de sistemas de acreditación, sanción y evaluación escolar y la descontextualización del contenido académico.

Otro aporte interesante lo realiza Dussel, (2006), en un artículo titulado De la primaria a la EGB: ¿Qué cambió en la enseñanza elemental en los últimos años?, incluido en una compilación titulada Diez miradas sobre la escuela primaria. La autora señala:

La escuela primaria en la Argentina se identificó con la escuela común y (...) se sostuvo como patrón de inclusión subordinada para amplias capas de la población, de escolaridad extendida y básica para todos. Además del currículum unificado (...) logró incluir a través de varias tecnologías, no siempre valoradas en su capacidad de impactar en la organización de la experiencia escolar. Una de ellas, el guardapolvo blanco, fue un modo importante de plantear el objetivo de una igualdad homogeneizadora. (Dussel, 2006, p. 97)

Justamente su investigación se abocará a la historia de los códigos de vestimenta en la escuela, lo que ha llamado el "régimen de apariencias escolar".

En la misma compilación, Terigi (2006) aludirá -indistintamente- a los "determinantes duros de las prácticas escolares" o "del dispositivo escolar" (Terigi, 2006, p. 197). La investigadora alertará sobre la persistencia de un formato escolar, y lo caracterizará, en pleno acuerdo con los desarrollos de Pineau (2001), por su estructura graduada y simultánea, la generación de una instancia colectiva para el aprendizaje y la particular organización espacial. Enfatizará en las 
consecuencias que dichos determinante tienen por un lado, sobre los desarrollos didácticos en los que maestros y profesores pueden apoyarse cuando diseñan sus propuestas de enseñanza y, por el otro, en la generación de llamado 'riesgo educativo', al aludir a fenómenos como la sobreedad escolar. Para esta autora la sobreedad es una situación relativa a la organización temporal de la escolarización, es decir, se transforma en un problema "en relación con el cronosistema que estructura la escolaridad primaria, tal como ésta viene desarrollándose históricamente" (Terigi, 2003, p. 2).

Precisamente esta autora investigará situaciones en las que el dispositivo escolar se modifica dramáticamente, como es el caso de los plurigrados de las escuelas rurales o los grados de aceleración (Terigi, 2002, 2006, 2007a, 2007b).

Lo propio hace Southwell (2009) al analizar escuelas de familia agrícola y escuelas de reingreso. Si bien se trata, al decir de esta autora, de dos nuevos formatos escolares del nivel medio, en estos estudios se vuelve evidente "que algunos componentes centrales del dispositivo escolar moderno se encuentran manifiestamente puestos en discusión y comienzan a instalarse formas de funcionamiento, dinámicas institucionales y funciones de lo escolar que difieren con algunas premisas centrales de aquél dispositivo" (Southwell, 2009, p. 3).

En dicho trabajo, la autora se pregunta "hasta qué punto podemos hablar de la escuela o lo escolar y remitir a un conjunto medianamente homogéneo de características cuando en realidad la diversificación de experiencias ha pasado a ser mucho mayor de lo que los formatos tradicionales incluían" (Southwell, 2009, p. 3). Asimismo señala que estos nuevos formatos de escuela, impulsados para resolver la exclusión educativa de los jóvenes que pertenecen a los sectores más pobres de la población, hacen evidente "la insuficiencia o la falta de pertinencia de aquella modalidad para atender a sectores clásicamente castigados y a nuevos fenómenos propios de la fragmentación y diferenciación social creciente" (Southwell, 2009, p. 5).

Finalmente, un grupo de trabajos referirán al papel performativo del dispositivo escolar. Baquero y Terigi (1996) enfatizarán en el tipo particular de aprendizaje que genera lo escolar: el aprendizaje escolar ${ }^{8}$. Lo caracterizarán como artificial y generador de "(...) efectos descontextualizadores sobre el desarrollo cognitivo (...)” (Baquero y Terigi, 1996, p. 1).

Otro efecto del dispositivo es la distribución de "posiciones subjetivas determinadas (...) las de alumno y docente (...)" (Baquero y Terigi, 1996, p. 7). Baquero (2001), por su parte, dedica todo un trabajo a vincular las concepciones del alumno con el dispositivo escolar.

El planteamiento de ese artículo pone en cuestión cierto discurso pedagógico que suele proclamar la necesidad de un desarrollo autónomo de los alumnos", al señalar que "la lógica del propio dispositivo escolar propone posiciones de fuerte dependencia, en parte, por la naturaleza inevitablemente asimétrica de los formatos escolares" (Baquero 2001, p. 2).

\footnotetext{
Los autores desarrollan más analíticamente este elemento del dispositivo que ya había sido señalado en Pineau (s. f.).

9 Sobre la fabricación escolar de un tipo de hombre 'autónomo', ver Lahire (2006).
} 
Finalmente, Corea (2000) sostiene como tesis que "en la situación educativa actual, el anacronismo del dispositivo pedagógico respecto de las nuevas figuras del niño genera violencia" (Corea, 2000, p. 1). La autora entiende la violencia como un desacople entre el lugar que el dispositivo pedagógico asigna a docente y alumno y el exceso, la diferencia, con que se presenta el niño actual respecto de ese lugar. Corea (2000) agrega que ese alumno que hoy llega a la escuela irrumpen en ella:

Irrumpe no sólo para violentar el lugar de "no ser" al que el dispositivo lo había confinado: irrumpe también para deslocalizar la figura del adulto-pedagogo que se había instituido en el dispositivo; irrumpe para deslocalizar el saber que sobre los niños el dispositivo había acumulado con paciencia (Corea, 2008, párr. 4) (...) el saber, tomado por la lógica de la información, se disemina en opiniones, pareceres, puntos de vista. Y la lógica de la información no requiere autorizados ni delegados; para ella no hay requisitos, ni saberes previos, ni escalafones. (Corea, 2008s, párr. 9)

Es decir, aquellos fenómenos connotados como "violencia" en la escuela serían, para esta autora, manifestación de la crisis actual de algunos de los elementos constitutivos del dispositivo escolar: el lugar del alumno, del saber y del docente.

Ahora bien, resulta interesante que ninguna de estas indagaciones tome como objeto de análisis las distintas experiencias de modificación de uno de los "determinantes duros del dispositivo escolar" (Terigi, 2006, p. 10) como son los programas de ampliación del tiempo escolar, los que, desde la década del 50, se registran en Argentina y han sido revitalizadas a inicios del siglo XXI por recomendaciones internacionales y la legislación educativa nacional.

\section{Conclusiones}

Hace más de dos décadas que asistimos en América Latina y en Argentina, en particular, al desarrollo de diferentes políticas orientadas a ampliar las jornadas escolares.

Entendemos que la implementación de estas políticas son oportunidades regias para analizar el comportamiento de la organización escolar ante los intentos de modificar uno de sus componentes duros, como lo es el tiempo; que constituyen laboratorios naturales que permiten analizar cuánto resiste la organización clásica del tiempo escolar, en qué cambia y cómo esos cambios (de ocurrir) implican a los restantes componentes de lo escolar (espacios, agrupamientos, etc.).

La revisión bibliográfica realizada nos indica que las investigaciones que han tomado el tiempo escolar y las distintas políticas y programas de extensión de la jornada escolar como 
objeto de indagación no dan cuenta de las vicisitudes que estas encuentran a la hora de intentar modificar los componentes duros del dispositivo escolar y, por otro lado, que las investigaciones que abordan como objeto de estudio el "dispositivo escolar" no han atendido las distintas experiencias de ampliación del tiempo escolar, aun cuando el tiempo es uno de los elementos constitutivos de dicho dispositivo.

Entendemos que lo expuesto justifica el desarrollo de investigaciones que analicen las formas en que se (re)organiza el dispositivo escolar en escuelas que implementen programas de extensión de la jornada escolar. Más específicamente se sostiene que describir la organización del espacio y del tiempo escolar en dichas escuelas, dar cuenta de la red que estos elementos configuran -es decir, de su funcionamiento como dispositivo-y analizar cómo la forma de (re) organización de dichos elementos del dispositivo; todo ello implica la de otros componentes de lo escolar: posición docente-alumno, agrupamientos y saberes.

\section{Referencias}

Administración Nacional de Educación Pública (ANEP). (2003). Resultados en escuelas de tiempo completo y escuelas de áreas integradas. Evaluación nacional de aprendizajes en Lenguaje y Matemática, 6to año enseñanza primaria, 2002 (Segundo informe). Uruguay: ANEP y PROYECTO MECAEP. Recuperado de http://www.oei.es/quipu/uruguay/eval lenguaje matematica.pdf

Agamben, G. (mayo-agosto, 2011). ¿Qué es un dispositivo? Sociológica 26(73), 249-264. Recuperado de http://www.revistasociologica.com.mx/pdf/7310.pdf

Aguerrondo, I. (1998). América Latina y el desafío del tercer milenio. Educación de mejor calidad con menores costos. PREAL, 10, 1-27. Recuperado de http://ww.thedialogue.org/ PublicationFiles/PREAL\%2010-Spanish.pdf

Ball, S. J. (Comp.). (1993). Foucault y la educación: disciplinas y saber. Madrid: Morata.

Banco Mundial. (2007). Uruguay. Equidad y calidad de la Educación Básica (Informe No 38082). Montevideo, Uruguay: Unidad de gestión del sector de desarrollo humano. Unidad de gestión de países para Argentina, Chile, Paraguay y Uruguay y Oficina Regional para América Latina y el Caribe. Recuperado de http://siteresources.worldbank.org/ INTURUGUAYINSPANISH/Resources/educacion.pdf

Baquero, R., Diker, G. y Frigerio, G. (Comps.). (2007). Las formas de lo escolar. Buenos Aires: Del Estante Editorial.

Baquero, R. y Terigi, F. (1996). En búsqueda de una unidad de análisis del aprendizaje escolar. En: Dossier Apuntes pedagógicos. Apuntes 2. Buenos Aires: UTE/ CTERA. 
URL: http://www.una.ac.cr/educare

Baquero, R. (2001). Las concepciones del alumno y el dispositivo escolar. Contextos de educación, 4(5), 156-165. http://www.unrc.edu.ar/publicar/cde/05/Baquero.htm

Belleï, C. (2009). Does lengthening the school day increase students' academic achievement? Results from a natural experiment in Chile [El alargamiento del día escolar ¿incrementa los logros académicos de los alumnos? Resultados de un experimento natural en Chile]. Economics of Education Review 28(5), 629-640.

Bermúdez, S. (s. f). Cultura escolar-cultura mediática. Apuntes para un encuentro. Buenos Aires: Programa Medios en la Escuela. Ministerio de Educación. http://estatico.buenosaires.gov. ar/areas/educacion/niveles/primaria/programas/medios en la escuela/culturaescolar.pdf

Bosco, A. (2000). Los recursos informáticos en la tecnología organizativa y simbólica de la escuela. Estudio de un caso. (Tesis doctoral inédita). Universidad de Barcelona, Departamento de Didáctica y Organización Educativa, España.

Centro de implementación de políticas públicas para la equidad y el crecimiento (CIPPEC). (2006). Estudio para la implementación de una política nacional de extensión de la jornada escolar. Buenos Aires: Autor.

Corea, C. (21 de agosto de 2008). El niño actual: Una subjetividad que violenta el dispositivo pedagógico. Jornadas sobre Violencia Social. [documento en blog tiempos posetatales]. Recuperado de http://postestatal.blogspot.com/2008/08/el-nio-actual-una-subjetividadque.html

Cotton, K. y Savard, W. G. (1981). Time Factors in Learning. Research on School Effectiveness Project: Topic Summary Report [Factores de tiempo en el aprendizaje. Investigación sobre el Proyecto de eficacia escolar: Reporte resumen del tema]. Portland, Oregon: Norhwest Regional Educational Lab. ERIC, ED 214706.

Department of Education USA (1994). Prisioners of Time [Prisioneros del tiempo]. Washington, DC: National Education Commission on Time and Learning. Recuperado de http://www2. ed.gov/pubs/PrisonersOfTime/TitlePage.html

Department of Education USA (1996). The Uses of Time for Teaching and Learning. Studies of education reform [Los Usos del Tiempo en la Enseñanza y el Aprendizaje. Estudios de la reforma educativa]. Washington, DC: Office of Educational Research and Improvement. Recuperado de http://www2.ed.gov/pubs/SER/UsesofTime/title.html

Diker, G. (2005). Los sentidos del cambio en educación. En G. Frigerio, G y G. Diker (Comps.). Educar: Ese acto político (pp. 127-138). Buenos Aires: Del estante editorial.

Dirección de Estudios Sociológicos de la Universidad Católica (DESUC). (2005). Evaluación jornada escolar completa (Informe final). Santiago de Chile: Pontificia Universidad Católica de Chile. Recuperado de http://www.opech.cl/bibliografico/Participacion Cultura Escolar/ Informe final jec.pdf 
Dussel, I. (setiembre-diciembre, 2003). Foucault y la escritura de la historia: Reflexiones sobre los usos de la genealogía. Revista Educación y Pedagogía, 15(37), 11-31. Antioquía: Universidad de Antioquia.

Dussel, I. (2006). De la primaria a la EGB: ¿Qué cambió en la enseñanza elemental en los últimos años? En F. Terigi (Comp.), Diez miradas sobre la escuela primaria (pp. 85-130). Buenos Aires: Siglo XXI Editores.

Escolano, A. (1992). Tiempo y educación. Notas para una genealogía del almanaque escolar. Revista de Educación, 298, 55-79. Recuperado de http://www.educacion.gob.es/dctm/ revista-de-educacion/articulosre298/re2980300486.pdf?documentld=0901e72b81357476

Fernández, M. (2000). La jornada escolar. Barcelona: Ariel.

Fernández, M. (abril, 2002). La jornada escolar continua en España: Dinámica y efectos. Conferencia presentada en Seminario Intemacional Complutense "Ritmos Psicológicos y Jomada Escolar". Madrid. Recuperado de http://es.scribd.com/doc/35952996/La-Jornada-EscolarContinua-SI-Ritmos-y-Jornada-UCM-2002

Foucault, M. (1997). Vigilar y castigar: Nacimiento de la prisión. Buenos Aires: Siglo Veintiuno.

Gimeno, J. (2005). La educación obligatoria: Su sentido educativo y social (3a ed.). Madrid: Ediciones Morata.

Gimeno, J. (2009). El valor del tiempo en educación. Madrid: Ediciones Morata.

Gvirtz, S. y Larrondo, M. (enero-diciembre, 2007). Notas sobre la escolarización. De la cultura material. Celulares y computadoras en la escuela de hoy. Teias, 8(14-15), 15-27.

Hargreaves, A. y Goodson, I. (2006). Educational Change Over Time? The Sustainability and Nonsustainability of Three Decades of Change and Continuity [¿Cambio Educativo a través del tiempo? La Sostenibilidad y la Falta de Sostenibilidad en Tres Décadas de Cambio y continuidad]. EAQ Educational Administration Quarterly, 42(1), 3-41. Recuperado de http:// eaq.sagepub.com/cgi/content/abstract/42/1/3

Husti, A. (1992). Del tiempo escolar uniforme a la planeación móvil tiempo. Revista de Educación, 298, 271-306.

Julia, D. (enero-junio, 2001). A cultura escolar como objeto histórico histórico [La cultura escolar como objeto histórico]. Revista Brasileira de História da Educação, 1(1) 9-43. Recuperado de http://www.rbhe.sbhe.org.br/index.php/rbhe/article/view/273/281

Lahire, B. (2006) El espíritu sociológico. Buenos Aires: Manantial.

Llach, J., Adrogué, C., Gigaglia, M. E. (Otoño, 2009). Do Longer School Days Have Enduring Educational, Occupational, or Income Effects? A Natural Experiment on the effects of 
lengthening primary school days in the city of Buenos Aires [¿Los días de clases más largos tienen efectos educativos, laborales o de ingresos duraderos? Un experimento natural sobre los efectos del alargamiento de los días de la escuela primaria en la ciudad de Buenos]. Economía, Journal of the Latin American and Caribbean Economic Association, 10(1), 81-114. Recuperado de http://muse.jhu.edu/journals/eco/summary/v010/10.1.llach.html

Ministerio de Educación República de Chile (agosto, 2003). Factores que inciden en el rendimiento de los alumnos. Prueba SIMCE $4^{\circ}$ Básico 2002. (Nota técnica). Santiago de Chile. Departamento de Estudios y Estadísticas. Recuperado de http://www.opech.cl/bibliografico/calidad equidad/Factores que inciden en el SIMCE 4 basico 2002 MINEDUC.pdf

Mitter, W. (1992). Tiempo escolar y duración de la enseñanza escolar en Alemania: Una comparación a nivel europeo. Revista de Educación, 298, 221-233.

Pineu, P. (2001). ¿Por qué triunfó la escuela? O la modernidad dijo: “Esto es educación”, y la escuela respondió: "Yo me ocupo". En P. Pineau, I. Dussel y M. Caruso (Comps.), La escuela como máquina de educar. Tres escritos sobre un proyecto de la modernidad (27-52). Buenos Aires: Paidós. 27-52. Recuperado de http://institutocieloazul.edu.ar/tspye/files/2011/08/ Pineau-Por-qu\%25C3\%25A9-triunf\%25C3\%25B3-la-escuela.pdf

Pineau, P. (s. f.). La escuela en el paisaje moderno. Consideraciones sobre el proceso de escolarización. Recuperado de www.histelea.unlu.edu.ar/pdf/pineau01.pdf

Querrien, A. (1994). Trabajos elementales sobre la escuela primaria. Madrid: Las Ediciones de La Piquea.

Rodríguez, C. (2009). El sentido del tiempo en las prácticas escolares. Revista lberoamericana de Educación, 49(1), 1-10.

Romero, C. A. (2006). Funciones del asesoramiento escolar en los procesos de cambio educativo en la sociedad del conocimiento: Un estudio de caso en escuelas secundarias de la ciudad de Buenos Aires. (Tesis doctoral). Universidad Complutense de Madrid. Facultad de Educación. Departamento de Didáctica y Organización Escolar. Madrid. Recuperado de http://www. ucm.es/BUCM/tesis/edu/ucm-t29346.pdf

Ruz, M. A. y Madrid, Á. (2005). Evaluación jornada escolar completa (Resumen ejecutivo). Santiago de Chile: Pontificia Universidad Católica de Chile. Dirección de Estudios Sociológicos. Recuperado de http://w3app.mineduc.cl/mineduc/ded/documentos/Resumen ejec evaluacion jec.pdf

Sapoznikow, W. (2008). La prescripción y la desviación: Programa; forma; gramática; cultura escolar y medios. XII Jornadas Nacionales de Investigadores en Comunicación. Nuevos escenarios y lenguajes convergentes. Escuela de Comunicación Social. Facultad de Ciencia Política y RRII Universidad de Rosario. Recuperado de http://www.redcomunicacion.org/ memorias/pdf/2008Saponencia\%20sapoznikow,\%20wendy\%20ok.pdf 
Southwell, M. (11-14 junio, 2009). La forma escolar desafiada: Escuela media, horizontes particulares y comunidades fragmentadas. Congress of the Latin American Studies Association, Rio de Janeiro, Brazil.

Terigi, F. (2002). Bases pedagógicas de los grados de aceleración. Buenos Aires: Secretaría de Educación del Gobierno de la Ciudad de Buenos Aires.

Terigi, F. (2003): La aceleración del tiempo y la habilitación de la oportunidad de aprender. Manuscrito no publicado.

Terigi, F. (Comp). (2006). Diez miradas sobre la escuela primaria. Buenos Aires: Siglo XXI Editores.

Terigi, F. (2007a). Enseñar en las "otras" primarias. El Monitor, 14. Buenos Aires: Ministerio de Educación de la Nación. Recuperado de http://www.me.gov.ar/monitor/nro14/dossier5. $\underline{\text { htm }}$

Terigi, F. (28-30 mayo, 2007b). Los desafíos que plantean las trayectorias escolares. En III Foro Latinoamericano de Educación Jóvenes y Docencia. La escuela secundaria en el mundo de hoy. Buenos Aires: Santillana.

Tyack, D. y Cuban, L. (1995). En busca de la utopía. Un siglo de reformas de las escuelas públicas. México: Fondo de Cultura Económica.

Universidad Nacional de Río negro. (2012). Programa de escuelas de jornada extendida: (re) organización del dispositivo escolar. Recuperado de http://www.unrn.edu.ar/sitio/index. php/informacion-institucional/comunicacion-institucional/articulos-de-divulgacion/2575programa-de-escuelas-de-jornada-extendida-re-organizacion-del-dispositivo-escolar

Valenzuela, J. P, Belleï, C., Osses, A. y Sevilla, A. (2009). Causas que explican el mejoramiento de los resultados obtenidos por los estudiantes chilenos en PISA 2006 respecto a PISA 2001. Aprendizajes y Políticas. Santiago de Chile: Secretaría Técnica FONIDE. Departamento de Estudios y Desarrollo, DIPLAP-MINEDUC. Recuperado de: http://centroestudios.mineduc. cl/mineduc/ded/documentos/F310843 Juan Pablo Valenzuela \%20Uchile.pdf

Varela, J. y Álvarez Uría, F. (1991). Arqueología de la escuela. Madrid: Las Ediciones de La Piqueta.

Varela, J. (1991). La maquinaria escolar. En J. Varela y F. Álvarez. Arqueología de la escuela. Madrid: Las Ediciones de La Piqueta.

Varela, J. (1994). Elementos para una genealogía de la escuela primaria en España. En A. Querrien (Ed.), Trabajos elementales sobre la escuela primaria (171-198). Madrid: Las Ediciones de la Piqueta.

Varela, J. (1995). Categorías espacio temporales y socialización escolar. Del individualismo al narcisismo. En J. Larrosa (ed.), Escuela, poder y subjetivación (pp. 155-189). Madrid: Las Ediciones de La Piqueta. 
Viñao, A. (2002). Sistemas educativos, culturas escolares y reformas. Madrid: Morata.

Von Koop, B. (1992). El tiempo escolar en el Japón. Revista de Educación, 298, 235-270.

Zorrilla, M., Langford, P., Ramírez, E. y García, L. (2008). Informe final de la evaluación del diseño del programa nacional de horario extendido en primaria. Programa nacional escuelas de tiempo completo. México: Universidad Autónoma de Aguascalientes. Recuprado de http://basica. sep.gob.mx/dgdgie/cva/sitio/pdf/evaluacionesext/PETC/2008/evaluaciones/informePETC. pdf

\section{Cómo citar este artículo, según APA:}

Vercellino, S. La ampliación del tiempo escolar: ¿Se modifican los componentes duros del formato escolar? Revisión bibliográfica sobre estas temáticas. Revista Electrónica Educare, 16(3), 9-36. Consultado de http://www.revistas.una.ac.cr/index.php/EDUCARE/issue/current

Nota: Para citar este artículo en otros sistemas puede consultar el hipervínculo "Como citar el artículo" en la barra derecha de nuestro sitio web:

http://www.revistas.una.ac.cr/index.php/EDUCARE/index 